Planetary Systems in the Universe - Observation, Formation and Evolution

Proceedings IAU Symposium No. 202, (C)2004 IAU

Alan Penny, Pawel Artymowicz, Anne-Marie Lagrange, 8 Sara Russell, eds.

\title{
What Photometric Space Telescopes Can Tell Us About Extrasolar Giant Planets
}

\section{S. Seager}

Institute for Advanced Study, Einstein Dr., Princeton, NJ 08540, USA

\section{Lam Hui}

Department of Physics, Columbia University, 538 West 120th St., New York, NY 10027, USA

\begin{abstract}
Within the next few years three microsatellites with part-permillion photometric capability (MOST, MONS, COROT) will be launched. These space telescopes, which were designed for asteroseismology, as well as other proposed or planned space telescopes to detect Earth-like transiting planets (Kepler and Eddington), will be able to observe the reflected light curves of the close-in extrasolar giant planets (CEGPs). The CEGPs are 0.05 AU from their parent star and are potentially bright in reflected light. For a transiting planet (at any orbital distance) moons and planetary rings may be detected.
\end{abstract}

\section{A New Class of Telescopes}

A number of planned or proposed space telescopes will be capable of part per million (ppm) photometry. In addition to their main goal of either asteroseismology or detecting transiting Earth-like planets, these space telescopes will be able to study small effects in extrasolar giant planets' (EGPs) reflected light curves or transit light curves.

Table 1. Photometric space telescopes with ppm capability. $\mathrm{A}=$ asteroseismology, $\mathrm{C}=$ CEGP light curves, $\mathrm{T}=$ Earth-like planet transits.

\begin{tabular}{llll} 
Satellite & Status & Main Goal(s) & Telescope Size \\
\hline MOST (Canada) & Launch 2002 & A, C & $15 \mathrm{~cm}$ \\
MONS (Denmark) & Launch 2003 & A & $34 \mathrm{~cm}$ \\
COROT (France) & Launch 2004 & A, T & $25 \mathrm{~cm}$ \\
Eddington (ESA) & ESA reserve mission & T, A & $1.2 \mathrm{~m}$ \\
Kepler (USA) & Proposed to NASA & T & $1 \mathrm{~m}$
\end{tabular}

\section{Photometric Light Curves}

The close-in EGPs (CEGPs), have $\sim 4$ day periods and are $\varsigma 0.05$ AU from their parent stars. The planet could be very bright in scattered light because of the proximity to the central star. The CEGPs phase varies as seen from Earth and will cause a small modulation in the combined star + planet light. For example, 


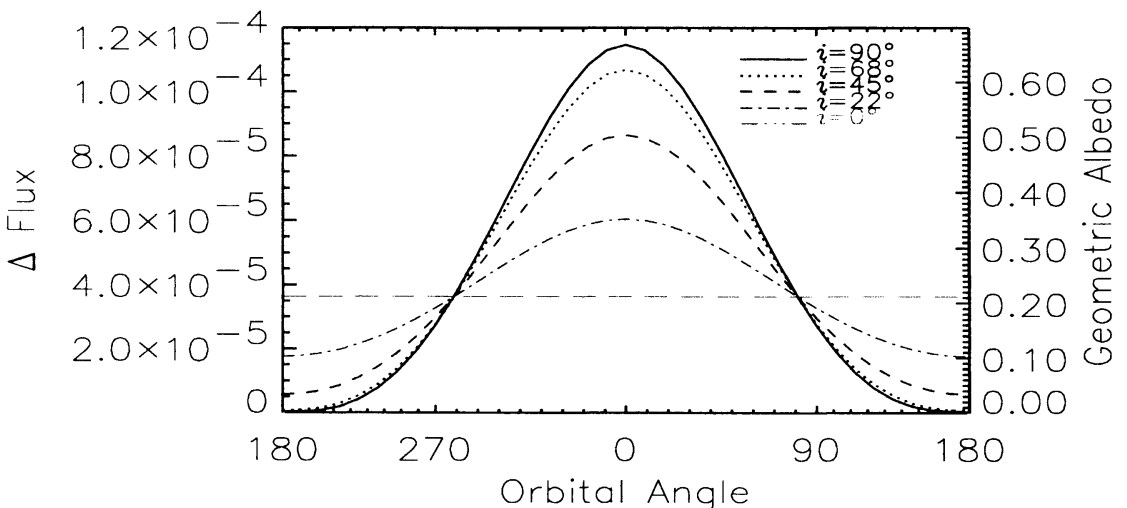

Figure 1. Lambert sphere light curve for different inclinations, for $R_{p}=1.4$ $R_{J}$ and $D=0.05 \mathrm{AU}$.

the Kepler mission proposal states they expect to find 870 CEGPs (with periods $<1$ week) from reflected light modulation. A Lambert sphere analytical estimate is shown in Figure 1. In reality the light curve will be smaller because of absorption, multiple scattering, and anisotropic scattering. The precise shape of the light curve depends on these parameters, which in turn depend on shape, composition, and size distribution of atmospheric particles. Figure 2 shows results from simulations of three atmospheres with different condensate types and sizes. While exact characterization of atmospheric particles would require polarization measurements or narrow band color photometry, general characteristics of the scattering particles can be derived from broad band light curves for extreme cases. See Seager, Whitney, \& Sasselov (2000) for more details.

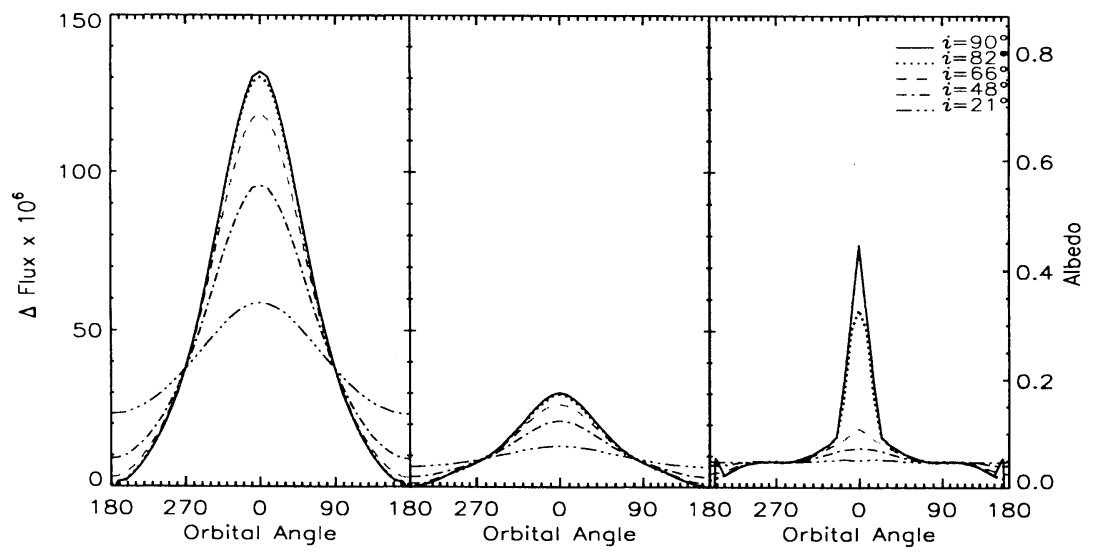

Figure 2. Simulation of $51 \mathrm{Peg}$ b assuming $R_{p}=1.2 R_{J}$ and $D=0.05 \mathrm{AU}$, for different particle types. Left: mean radius $(\bar{r}) 0.01 \mu \mathrm{m}$ for $\mathrm{MgSiO}_{3}$; center: $\bar{r}=0.1 \mu \mathrm{m}$ of a homogeneous mix of $\mathrm{MgSiO}_{3}, \mathrm{Al}_{2} \mathrm{O}_{3}$, and $\mathrm{Fe}$; right: same as center but with $\bar{r}=10 \mu \mathrm{m}$. 


\section{Moons and Rings}

Large moons can be found by their transit light curve or from the time shift of planet transits due to the planet-moon barycentric motion (Sartoretti \& Schneider 1998). Rings may also be detected during occultation depending on their size and orientation with respect to the stellar disk. A single opaque ring $1 / 2$ the size of Saturn's rings for a CEGP with $R_{p}=1.4 R_{J}$ orbiting a sun-like star would deepen the planet transit light curve by $0.05 \%, 0.5 \%, 1 \%, 1.2 \%$ for inclinations of the planet's equator to orbit $86^{\circ}, 60^{\circ}, 40^{\circ}, 20^{\circ}$ respectively. Rings will also change the shape of the transit light curve. (See also Charbonneau et al. 2000.) Rings may also produce a detectable signal from scattered stellar light.

\section{Star Spots}

Photometric variation may be caused by the occultation of a small part of a nonuniform stellar disk. One example is star spots. Below are theoretical transit light curves of a CEGP and Earth-like planet crossing an Earth-sized star spot on the stellar disk. The spot occultation duration is related to planet size and the magnitude of the effect is related to the spot-planet ratio.
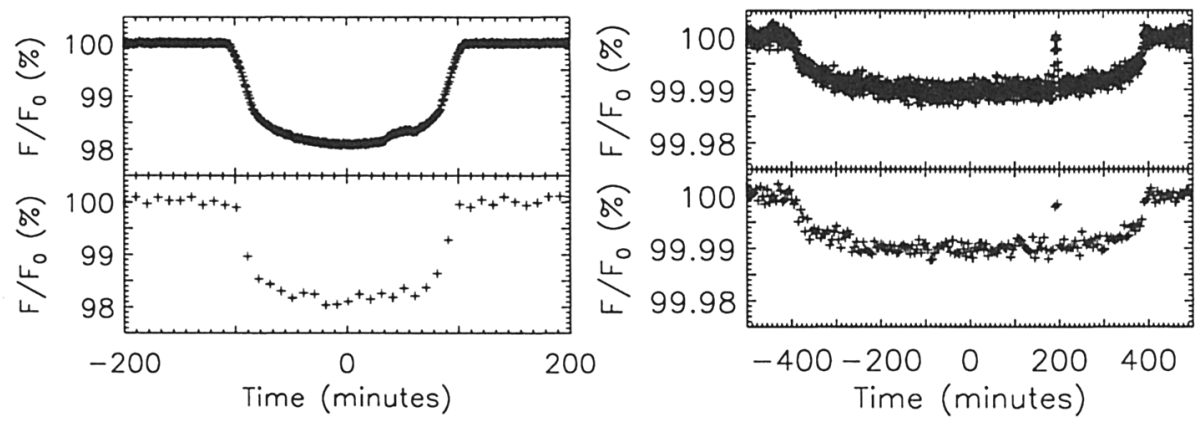

Figure 3. Left: CEGP transit. Top: 1 min resolution, $10^{-4}$ level Gaussian noise; bottom: $10 \mathrm{~min}$ resolution, $8 \times 10^{-4}$ level noise. Right: Earth-like planet transit. Top: $1 \mathrm{~min}$ resolution, $10^{-5}$ level noise; bottom: $5 \mathrm{~min}$ resolution, $10^{-5}$ level noise.

Acknowledgments. We thank Barbara Whitney and Dimitar Sasselov for useful contributions. SS is supported by the W. M. Keck Foundation.

\section{References}

Charbonneau, D., Brown, T. M., Gilliland, R. L., Noyes, R. W., \& Burrows, A. 2000, in ASP Conf. Ser. xx, Planetary Systems in the Universe, ed. A.J. Penny, P. Artymowicz, A.-M. Lagrange, \& S.S. Russell, (San Francisco: ASP), in press

Sartoretti, P., \& Schneider, J. 1998, A\&AS, 134, 553

Seager, S., Whitney, B. A., \& Sasselov, D. D. 2000, ApJ, 540, 504 